\title{
Discussion on application of VR technology in physical teaching
}

\author{
Jiangjunzhe Tang*, Chu Wang, Jinwei Liu, and Daiping Sun \\ College of software, Jilin University, Changchun136000, China
}

\begin{abstract}
In an age of further development of information, but the old teaching model is out of fashion and cannot be applied to current production and learning. In order to keep pace with changes, the old teaching model needs to be updated, while VR technology has powerful function of virtual simulation, which is applied in the field of physical teaching attracts public attention. This paper describes the great potential of VR technology applied in education, and also points out the current problems in VR education and the prospects for VR technology, in order to promote physical teaching with the support of VR technology.
\end{abstract}

\section{Preface}

Since Facebook purchased Oculus in 2014, virtual reality technology (VR) has gradually begun to attract public attention. In 2016, virtual reality technology appeared as an absolute protagonist in the International Consumer Electronics Show (CES), which showed the great potential of VR technology around the world. At the same time, various industries have begun to actively carry out VR application research, hoping to make full use of its value. Due to the popularity of VR technology, some people also called 2016 "VR first year". The potential of VR technology makes many manufacturers to believe that it will likely become next-generation computing platforms instead of $\mathrm{PC}$ and smart phones [1].

In an age when such a new technology continues to emerge, the original teaching model cannot be applied to the more developed society of information technology. Therefore, in order to change the original teaching model, the government issued a series of policies to promote the construction of educational information in China. This paper will discuss the application of VR technology in middle school physical teaching.

\section{Overview of VR Technology}

VR technology is new technology in the computer field, which combines computer technology, multimedia technology, image technology, simulation technology, and a variety of electronic technologies. It uses computers to build a three-dimensional virtual world, where users can use input devices to interact with the virtual world and feed back to users through multiple sensors and output devices [2]. Generally speaking, technology constructs a virtual world where users can perform any operation that can achieve the same

*Corresponding author: tjjz3415@mails.jlu.edu.cn 
result with the real world. The reason why application of VR technology has become the focus of public attention is such a special characteristic.

\section{VR TECHNOLOGY IN MIDDLE SCHOOL PHYSICAL TEACHING}

As an experiment-based subject, physical abstraction often makes it difficult for some students to understand [3]. However, in the middle school stage, most students just get in touch with the physics. It is particularly important to cultivate interest in physics in this key point, and it also lays a good foundation for further learning. However, not all students can learn the laws of physics directly from the textbooks and the teacher's experiments in class. Some reasons, such as the incomplete construction of the laboratory, make it difficult for every students to implement experiments. As a result, it is appropriate to apply the powerful functions of VR technology into the physical teaching [3]. Constructing virtual scenes through VR technology allows students to observe and to learn in virtual scenes, which is of great help to the improvement of teaching quality.

\subsection{Application of VR Technology on the Explanation}

Different from the way of using multimedia to play video to explain knowledge in the past, the VR technology is applied to the lectures, which represents the knowledge points in the virtual world with corresponding physical phenomena. Meanwhile, the students can use the head display to observe physical phenomena from multiple dimensions, which is intuitive and easy for students to understand the laws of physics. For example, the students can watch celestial movements in the virtual world, and observe how they rotate and revolve, and in which plane thy move and also can observe the composition and movement of molecules, atoms and so on. Through vivid demonstrations, students can more profoundly understand the laws of physics and at the same time, they are also easy to remember. It is difficult to achieve with PPT teaching, but it can be done with a few minutes of VR video. This is also the initial stage of applying VR in teaching.

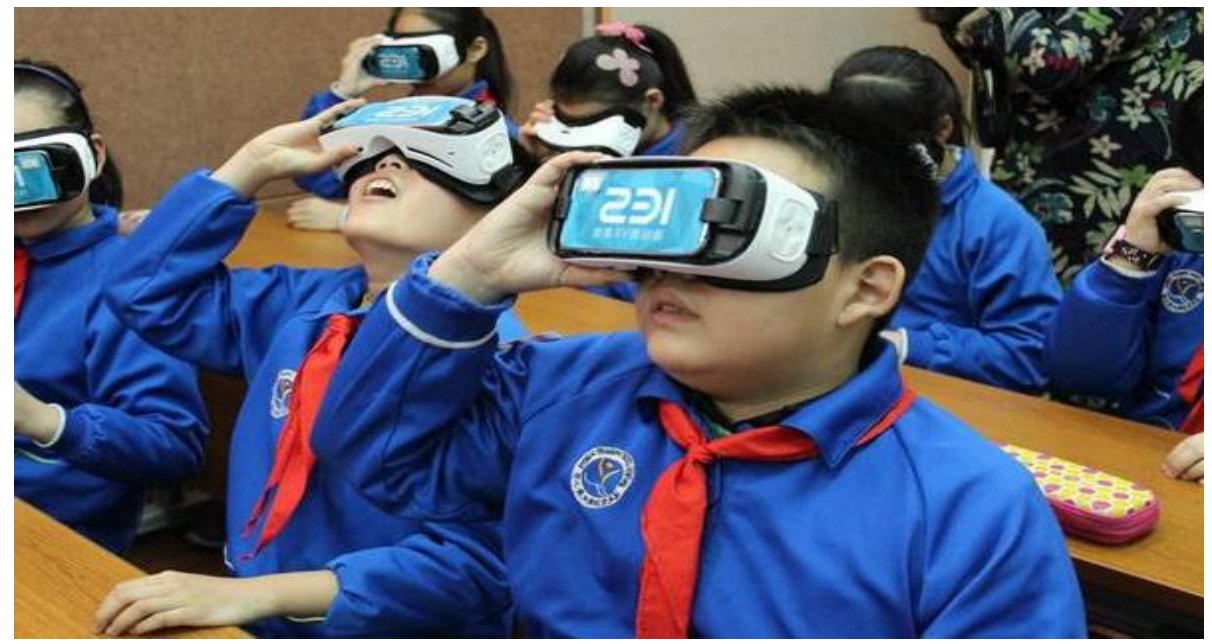

Fig. 1. Students use VR glasses to watch the teaching content. 


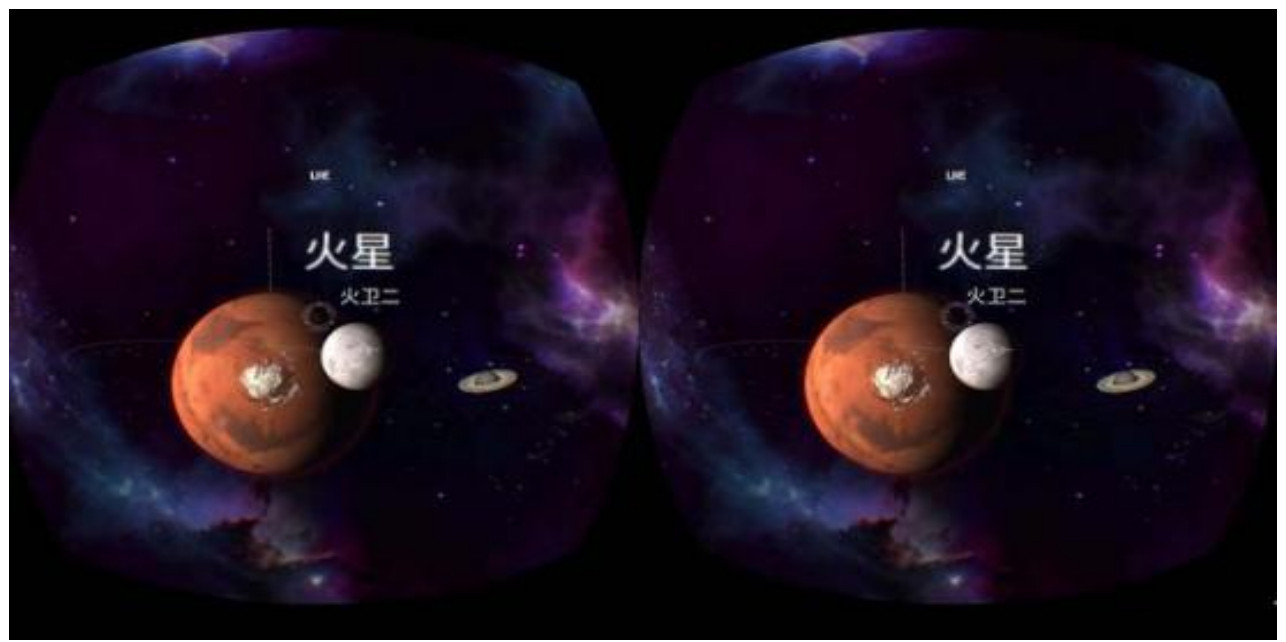

Fig. 2. Scenes seen in VR glasses.

Figure 1 shows some startup companies' VR teaching attempts in primary and secondary schools. At present, due to high equipment costs and less application software, only VR video can be used for teaching. As shown in Figure 2, students can use teaching videos that VR equipment can see. Through VR teaching videos, they can observe physical phenomena more clearly and objectively.

\subsection{VR Technology Application in Experiment}

It is to maximize the use of VR technology's function to be applied in physical experiments. The physics will lose the soul without the experiments. People could use VR technology to build a virtual laboratory including a variety of virtual equipment. In this laboratory, users can use any equipment "irresponsibly" and randomly, and conduct any experiments. Even experiments that are extremely difficult to implement such as nuclear reactions can be conducted there. In addition to such an interesting use, it is more important to achieve the teaching purpose. Students can learn to do experiments correctly in the laboratory with using VR equipment, and observe the experimental phenomena, analyze the data and get the physical laws. At the same time, students can also observe some abstract movements by giving corresponding conditions, such as students can arbitrarily place a charge anywhere in the composite field and give the velocity in any direction to observe how the charge moves. This kind of teaching model like playing games can stimulate students' enthusiasm and reach to "happy teaching". However, there is still a big difference between VR technology application in experiment and VR technology application in explanation. The application in explanation as long as the provision of quality teaching video can be completed, but the application in experiment teaching needs to meet the following requirements:

First, the virtual experimental results must be the correct result. This is also the most important point. If students get false results through virtual experiments, virtual experiments will become meaningless. In order to achieve this goal, we need to provide higher quality scripts. It is difficult to stimulate reality using the physics engines of some development platforms.

Second, models are highly simulated. A good model can give users a better experience and be more immersed in it. For example, in the balance experiment, it is necessary to adjust the rider code and the balance nut. If the model is not sufficiently emulated and not 
elaborate enough, the purpose of the experimental operation teaching cannot be achieved. Figure 3 shows the balance of the real world. In Figure 4, we use 3d Max to create the same proportion of the simulation model. Only realistic models can give the user enough immersion.

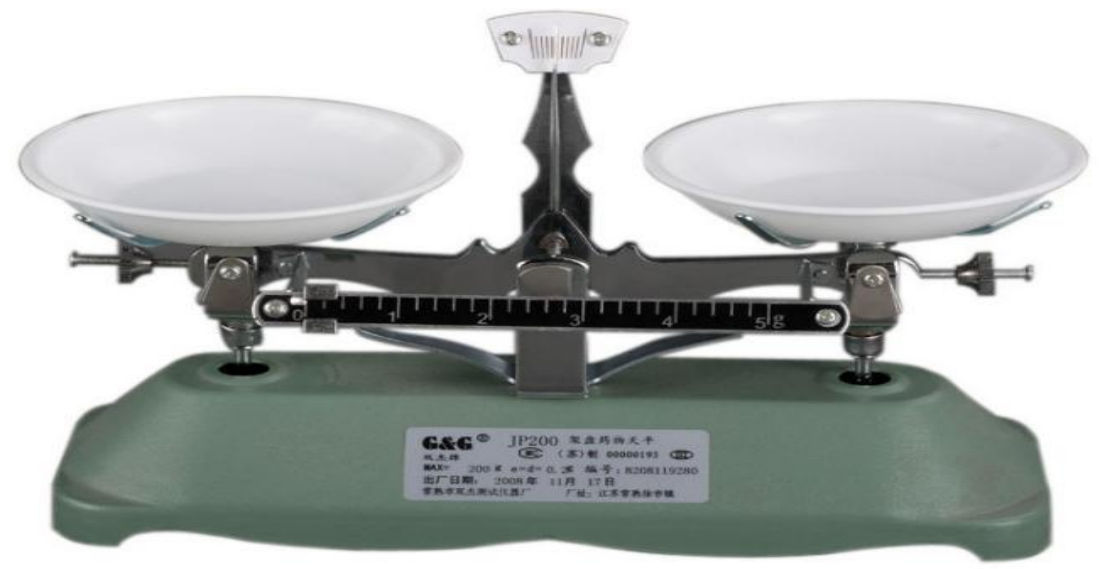

Fig. 3. Balance in Reality.

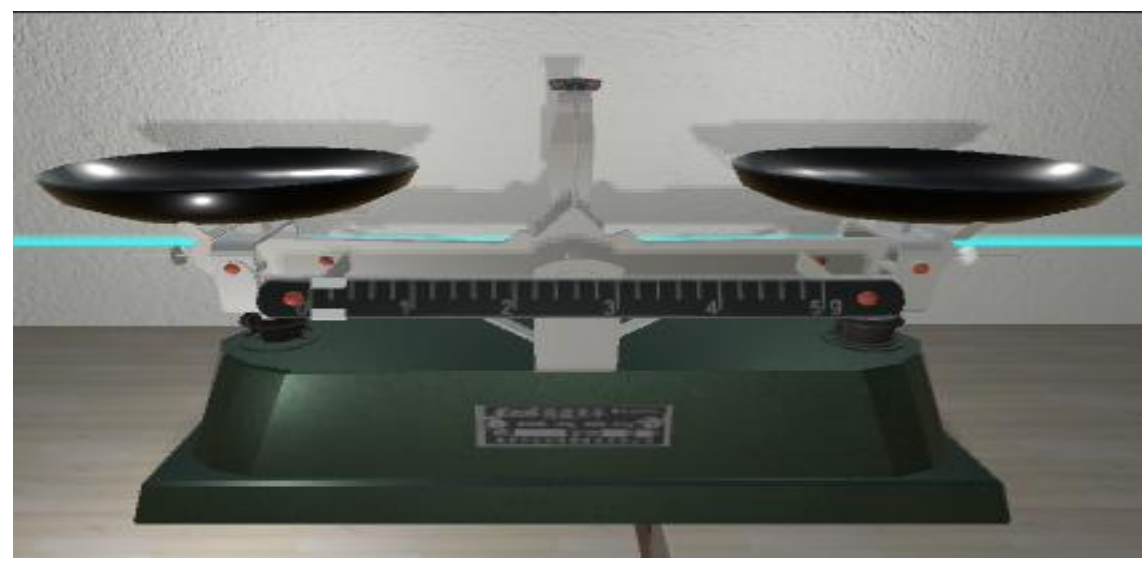

Fig. 4. Balance model made with 3d Max.

Third, it gives students more autonomy. Can improve its practical operation ability, provide all equipments that may be used in the virtual laboratory, and give the corresponding experiment scheme, let the student autonomously experiment.

Using VR technology to carry out experiments, basically all experiments in middle school can be done by using only VR equipment and computers. Not only is it not limited by time and space, the experiment method is flexible and diverse, which is conducive to maintaining the enthusiasm of students and improving teaching quality and efficiency.

\section{Problems and Prospect of "VR + Education" in the Current Stage}

As a new thing, "VR+ education" does have inestimable power, and some new pioneering companies actively promote it. For the moment, there are still many practical problems in the application of VR in the field of education, such as expensive equipment and less 
application. All these reasons make most schools reluctant to have a try. It is difficult to popularize them in a short time[4]. Because the model simulation is not enough and the virtual world environment is too ideal, some people think that the results in virtual reality cannot be applied to reality. Even if the virtual world infinitely gets close to the real world, it cannot replace the reality.

However, VR technology's powerful function of virtual simulation cannot be replaced by any technology. It is believed that the cost of hardware equipment will become lower and lower, with the great development of technology. Sooner or later, it will spread to every household. The simulation can also use a large amount of data to collect and input through a script, making the virtual world infinitely get close to reality. Even though it can not be "replaced", it can also make users familiar with the operation process, and thus improve the successful rate in the real world. Because the concept of VR is easy to understand and the virtual world is similar to reality, people are more likely to accept this technology.

In short, any technology cannot perfectly meet all the requirements of the users [5]. Only they make better use of technology, can they maximize the benefits.

\section{References}

1. Yu Cao, Virtual Reality: The Next Generation Computing Platform You Must Know,5-20(2016)

2. Ilana Dubovi,Sharona T. Levy,Efrat Dagan,Computers \& Education, 113,16-27(2017)

3. Huiying Yao,Education and profession, 5,144-145(2011)

4. JinGen Yuan,ZhiGuo Li, Applied Mechanics and Materials,644-650,5594-5597(2014)

5. Maja Pivec,Organizacija,45,125-130(2012) 